\title{
Cross Cutting Structural Design for Exploration Systems
}

Edmund B. Semmes

George C. Marshall Space Flight Center, MSFC, Alabama 35812

$\begin{array}{ll}\text { Al } & =\text { Aluminum } \\ \mathrm{cSv} & =\text { centiseviert } \\ \mathrm{CEV} & =\text { Crew Exploration Vehicle } \\ \text { ECLSS } & =\text { Environmental Control \& Life Support Systems } \\ \text { FAST } & =\text { Functional Analysis Systems Technique } \\ \text { HW/SW } & =\text { Hardware/Software } \\ \text { IPT } & =\text { Integrated Product Team } \\ \text { LEO } & =\text { Low Earth Orbit } \\ \text { LH2 } & =\text { Liquid Hydrogen } \\ \text { LOX } & =\text { Liquid Oxygen } \\ \text { LCD } & =\text { Liquid Crystal Display } \\ \text { MCA } & =\text { Mass Constituent Analyzer } \\ \text { MMOD } & =\text { Micrometeroid and Orbital Debris } \\ \text { MSFC } & =\text { Marshall Space Flight Center } \\ \text { NCRP } & =\text { National Council on Radiation Protection \& Measurements } \\ \text { TCS } & =\text { Thermal Control System } \\ \text { TRL } & =\text { Technology Readiness Level } \\ \text { VSE } & =\text { Vision for Space Exploration }\end{array}$

\section{Introduction}

II. Functional Analysis Systems Technique (FAST)

III. A Multifunctional Structural System

IV. Conclusion

Appendix - Multifunctional Structural Wall System Technology Requirements

\section{Acknowledgements}

References

\section{Introduction}

The challenge of our new National Space Policy and NASA's Vision for Space

Exploration (VSE) is keyed to the development of more effective space access and transportation systems. Optimizing in-space systems through innovative cross cutting structural designs that reduce mass, combine functional requirements and improve performance can significantly advance spacecraft designs to meet the ever growing demands of our new National Space Policy. Dependence on limited structural designs is no longer an option. We must create robust materials, forms, function and evolvable systems. We must advance national policy objectives in the design, development, test and operation of multi-billion dollar new generation crew capsules by enabling them to evolve in meeting the requirements of long duration missions to the moon and mars.

This paper discusses several current issues and major design drivers for consideration in structural design of advanced spacecraft systems. Approaches to addressing these 
multifunctional requirements is presented as well as a discussion on utilizing Functional Analysis System Technique (FAST) in developing cross cutting structural designs for future spacecraft. It will be shown how easy it is to deploy such techniques in any conceptual architecture definition or ongoing preliminary design.

As experts in merging mission, safety and life support requirements of the frail human existence into robust vehicle and habitat design, we will conquer the final frontier, harness new resources and develop life giving technologies for mankind through more innovative designs. The rocket equation tells us that a reduction in mass optimizes our propulsive results. Primary and secondary structural elements provide for the containment of gases, fluids and solids; translate and sustain loads/impacts; conduct/radiate thermal energy; shield from the harmful effects of radiation; provide for grounding/bonding of electrical power systems; compartmentalize operational functions; and provide physical interface with multiple systems.

How can we redefine, combine, substitute, rearrange and otherwise modify our structural systems to reduce mass? New technologies will be needed to fill knowledge gaps and propagate new design methods. Such an integrated process is paramount in maintaining U.S. leadership and in executing our national policy goals. The cross cutting process can take many forms, but all forms will have a positive affect on the demanding design environment through initial radical thinking. The author will illustrate such cross cutting results achievable through a formal process called FAST. The FAST example will be used to show how a multifunctional structural system concept for long duration spacecraft might be generated.

\section{Functional Analysis Systems Technique (FAST)}

As described, functional analysis has been a critical aspect of product development and systems engineering for many decades. Spacecraft developers have used such logical techniques in every major aerospace endeavor even if only subconsciously. It defines the very heart of the systems engineering process and forms the bridge between requirements and design synthesis. It can at times be an unsettling form of analyses because of its fundamental divergent character. The question of how, why and when of requirements or products can be bluntly revealing when done properly. However, the elimination of biases and organizational barriers during IPT generated functional analysis opens up a powerful door to creativity and problem solving. It is this high intensity creativity and teamwork that is critical to robust spacecraft developments in the future. In their characterization of the current launch vehicle design process, Blair, et.al. ${ }^{1}$ espouse the need to continuously improve design processes through more effective idea stimulation approaches, seamless integration of functional analyses and direct synthesis methods for concept identification. An intensive and repetitive functional analysis process is the key to achieving these goals within realistic time constraints.

The Functional Analysis Systems Technique (FAST) was introduced by an engineer at Sperry Univac (Unisys) in the 1960's named Charles W. Bytheway. FAST is a methodology to decompose basic function and organize it into a logic diagram providing insight and opportunity for creative new ways of accomplishing functions. The first step 
in the methodology is to identify the mission of the system while bounding the scope of the problem, or opportunity. This can be done in several ways to generate the informational parameters of the product and generally educate the entire IPT. Next, the use of brainstorming can generate a long list of functions to accomplish the objective or mission developed in the first step. These are listed in the middle column of a three column matrix with the adjacent columns representing "WHY" and "HOW," as columns one and three, respectively. This matrix facilitates the construct of the final FAST diagram where Postitß notes can be used to align the logic flow of the model. Many times it is during this stage that a new higher order function or objective is perceived and becomes a defining moment for the team. Once the objective or higher order function is established it is placed to the far left of the chart. The question, "How is this function performed?" is asked and this becomes the "basic function" of the product. The process continues moving to the right defining all dependent functions, independent functions (above the critical path) and activities (below the critical path).

All future spacecraft designs will include some sort of structural wall system and, as such, represents an excellent choice to illustrate the power behind the FAST technique. The following chart illustrates the results of a small team of MSFC engineers coming together to critically analyze the structural wall system with the intent of broadening its functional primacy, eliminating the mass in other subsystems and optimizing its integrated form (See Figure 1).

\section{Structural Wall System Functional Analysis}

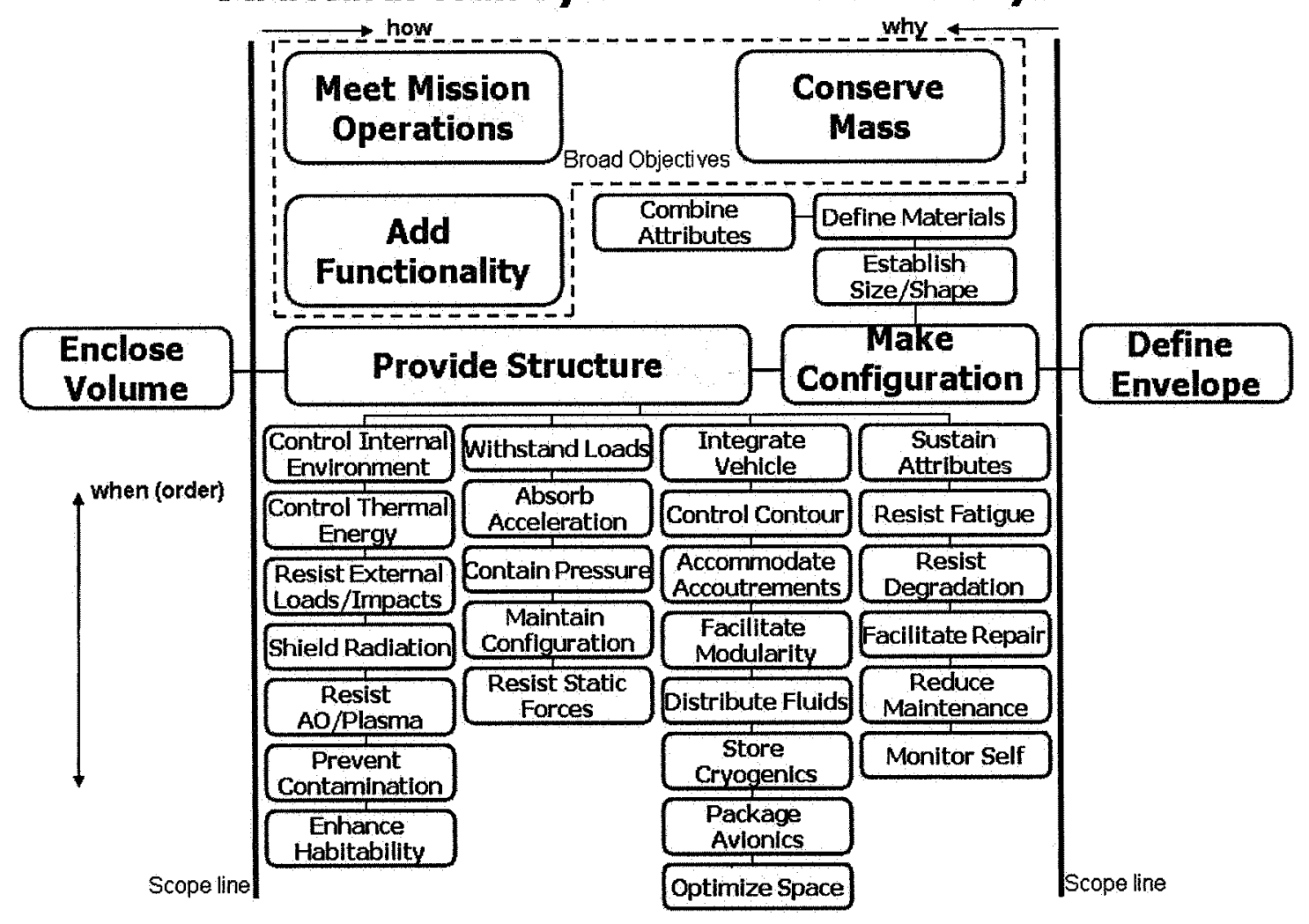

Figure 1 - Example of Structural Wall System FAST Diagram 
The hierarchical decomposition methods of the FAST opened the opportunity for creativity in adding functions typically allocated to other subsystems. For instance, distribution of fluids is typically allocated to TCS and ECLSS subsystem design processes. Foundational in the realization of more effective systems is the upfront challenge of typical functional allocations to cross cut into structural design. In the case of cross cutting distribution of fluids, successful integration into a structural form can lead to the elimination of mass and the complexities associated with conventional conduits, fittings and brackets.

Shielding for radiation is illustrated as a critical example of expanding functionality or combining functions to achieve more efficiency in design. To illustrate more clearly how functional combinations and integration can be achieved it is necessary to utilize FAST on each added function, such as "Distribute Fluids" or "Shield Radiation" (See Figure 2). The successively lower ordered functions serve as common building blocks needed to synthesize an integrated, effective design. For instance, "polymerize materials" and "provide mass" might be combined with other structural wall system functions such as "absorb acceleration" and "resist impacts" when defining the structural wall materials and configuration.

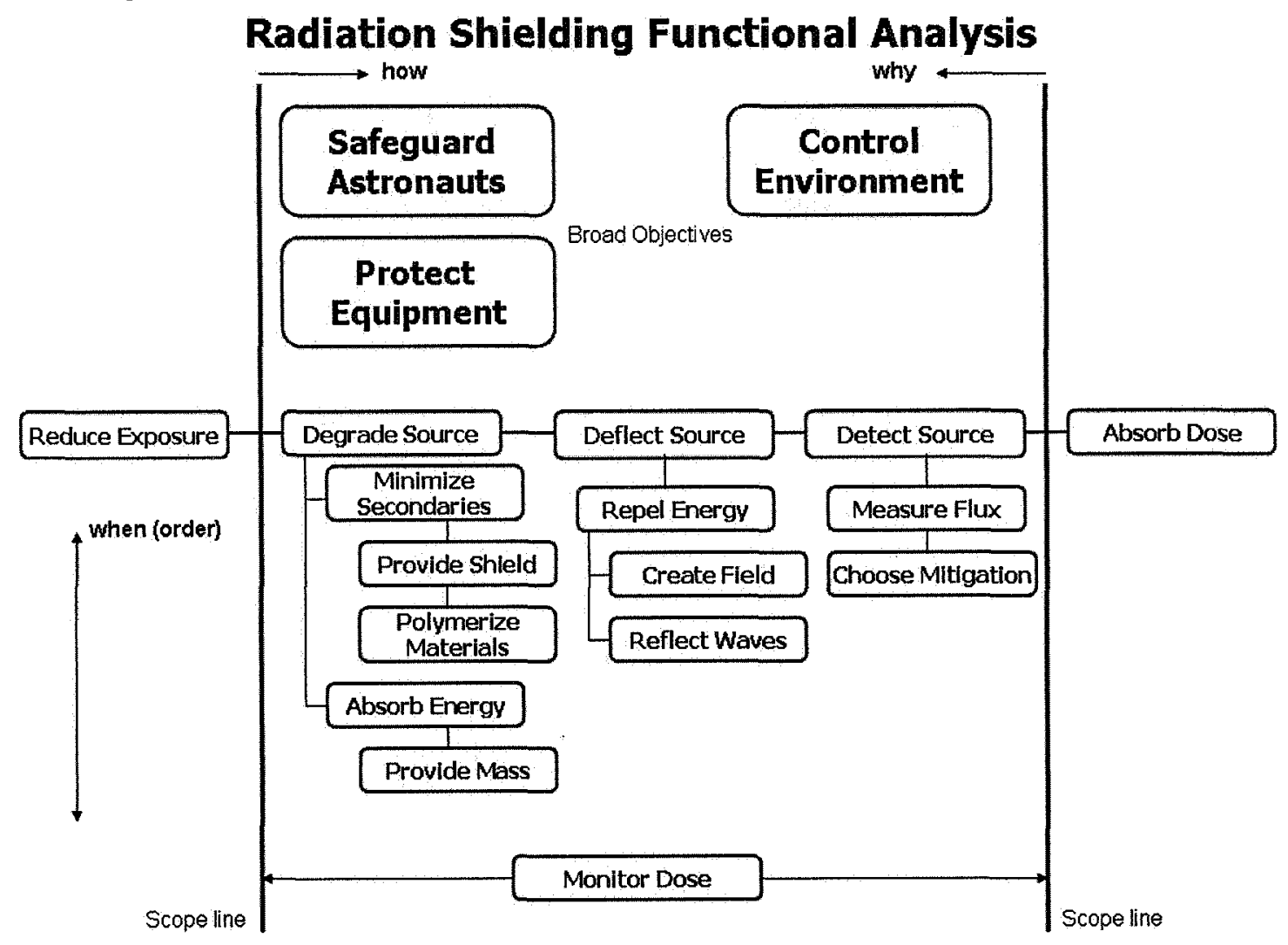

Figure 2 - Example of Radiation Shielding FAST Diagram

At the most fundamental level of every intended function, the FAST process naturally results in the elimination, combination, simplification and economy of functions when performed in a disciplined fashion with experienced IPT design team members. The 
information, creativity, analysis, development and presentation phases of FAST provide a logical framework for communication with the customer, responsive design synthesis and effective problem resolution.

The feedback available from FAST has an additional benefit in that it can directly help align technology development initiatives with strategic need. George and Powers ${ }^{3}$ report, "Design changes are far cheaper and more cost effective at low TRL than after a technology has matured in a direction that is not well aligned with the end application." Any R\&D study, grant, technology project or program that cannot rationalize its requirements, is a luxury no organization can afford. Cook and Tyson ${ }^{4}$ highlight in "Next Generation Launch Technology Program Lessons Learned" that "systems analysis should be used to guide and assess technology development, beginning early in the program. Systems analysis should develop quantifiable priorities and requirements against which technology projects execute." Nothing is more fundamental to successful product development and the evolution of our built environment. The wisdom to discern utilization of new and old technologies is an experiential based process resulting in the establishment of priorities. These judgments are in vain absent top to bottom systems analysis that clarifies organizational/mission/program objectives. Figure 3 shows the relationship of the VSE with strategic planning, requirements development, allocation and the functional analysis process as it defines HW/SW and technology deployments.

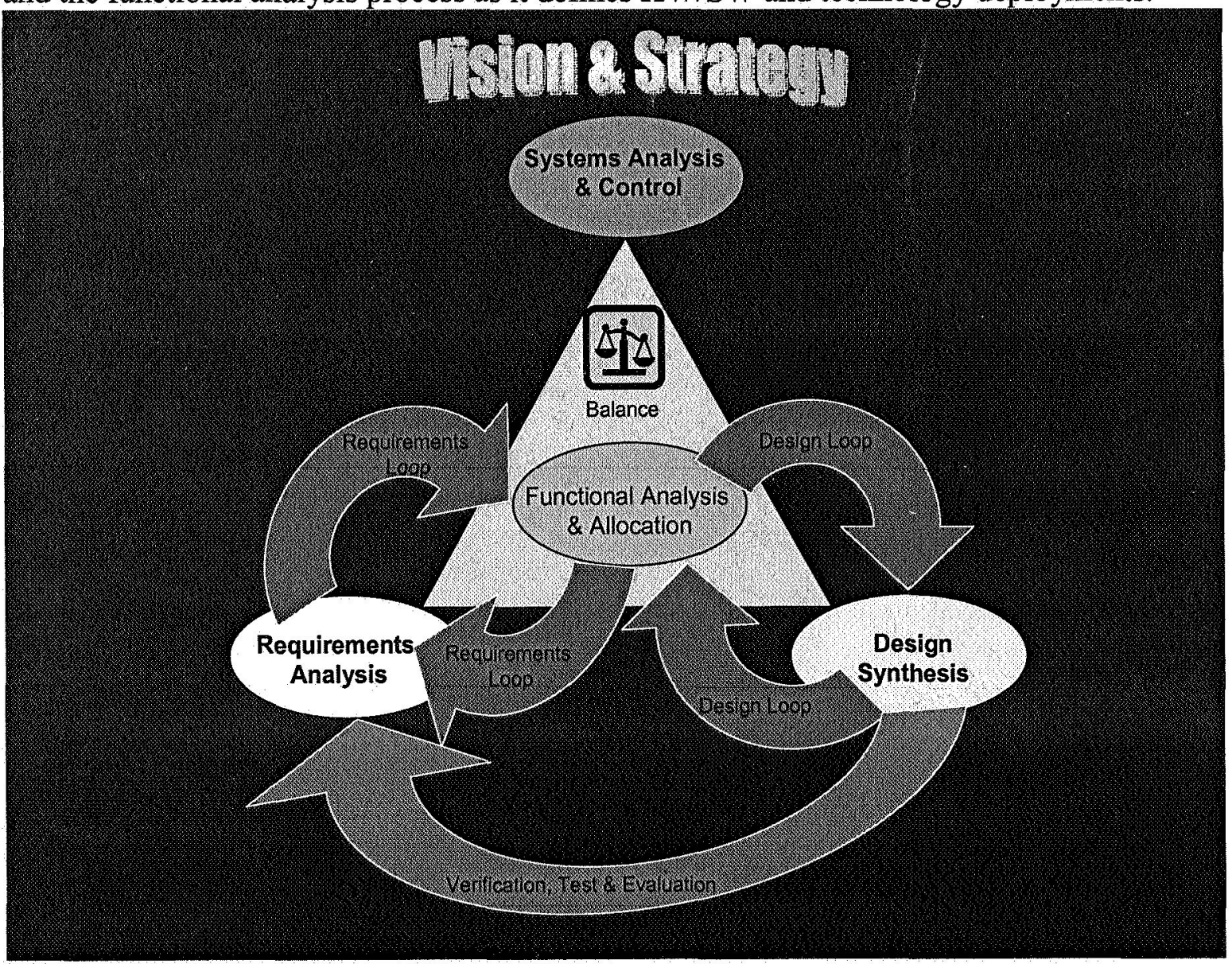

Figure 3 - Relationship of Vision/Strategy with the Systems Engineering Process 
This overall structure is critical in managing expectations of our stakeholders and at the core of it all is functional analysis. The rigor of effective functional analysis and its outcomes should never be a threat to public policy makers as it enables the policies established with physical form.

\section{Multi-functional Structural System}

Long duration spacecraft design for the human exploration of space will necessitate fundamental design attributes that drive the final form, fit and function of vehicles, habitats, landers and other manned spacecraft. No less fundamental than the Wright brothers development of design principles of atmospheric flight, the design principles for long duration manned space missions must evolve from a vision or concept of safe human transportation in a harsh space environment, with little or no infrastructure support and a resource constrained condition. An optimized solution will necessitate the development of new design tools and techniques never before used.

NASA's manned spaceflight program has historically been limited to the Earth's near neighborhood consisting of relatively short duration manned missions. Consequently, mass, habitable volume, power and radiation shielding have not presented unsurmountable design issues. Long duration missions to the moon and Mars will significantly surpass the design techniques of the past. Architectural and human factors considerations will greatly influence the extent to which individuals and groups can live and work together comfortably and productively. ${ }^{9}$ An intensively integrated and robust set of design tools will be needed to assess a multitude of mission scenarios, operational techniques and sustainability options. For instance, integrated vehicle health systems can no longer be a follow-on design, but an upfront requirement that influences hardware and software design. All functional vehicle design disciplines will have to align with these attributes that drive form, fit and function of new spacecraft.

The discussion of these cross cutting structural design techniques for exploration is facilitated by specific information, examples of the methods and potential outcomes. As shown, a truly multifunctional structural system has great potential for exploration systems whether performed at the box level or integrated mission level. The "art" becomes the ability to define the form of all these complex, integrated functions. Our example extends the work of our structural wall system design for a manned exploration craft with long duration missions "beyond LEO."

The remainder of this paper will illustrate how the addition of cross cutting functions to the structural wall system preserves mass, volume, resources and better integrates the manned spacecraft example. Radiation shielding, fluids and electrical distribution functions will be discussed in detail to directly show how the integration process begins.

\section{Radiation Shielding}

Safety is NASA's highest priority. Safety is the freedom from those conditions that can cause death, injury, occupational illness, damage to or loss of equipment or property, or damage to the environment. Exposure of astronauts to energetic solar and galactic cosmic rays is a major concern for long duration crewed missions in deep space (beyond 
Earth's magnetosphere) and in higher inclination LEO missions. The effects of materials and structures on the internal radiation environment will be a constraint on the design of future spacecraft and habitats. The major goal is to enable human exploration of space without exceeding limits on risks from space radiation. Space radiation is distinct from terrestrial forms of radiation, being comprised of high-energy protons and heavy ions and the secondary particles produced during fragmentation as the particulate radiation passes through matter. NASA supports research that emphasizes studies of the basic, as well as applied physical aspects of the interaction of high energy and highly charged particles with matter, and the design, fabrication, and testing of multifunctional radiation shielding materials. Like many space exploration challenges, the complexities create a vital need for a multi-disciplined approach.

The human exploration of space will require shielding alternatives for very long duration missions. As an example, consider the time required for a mission to Mars. Estimates vary from 661 to 905 days $^{5,7}$ total mission times' dependent on mission design and trajectory assumptions. Figure 4 illustrates the effects of current safe dose requirements in LEO for shielding materials applied to a two year "notional" Mars mission limit (safe limits have not been established for deep space missions).

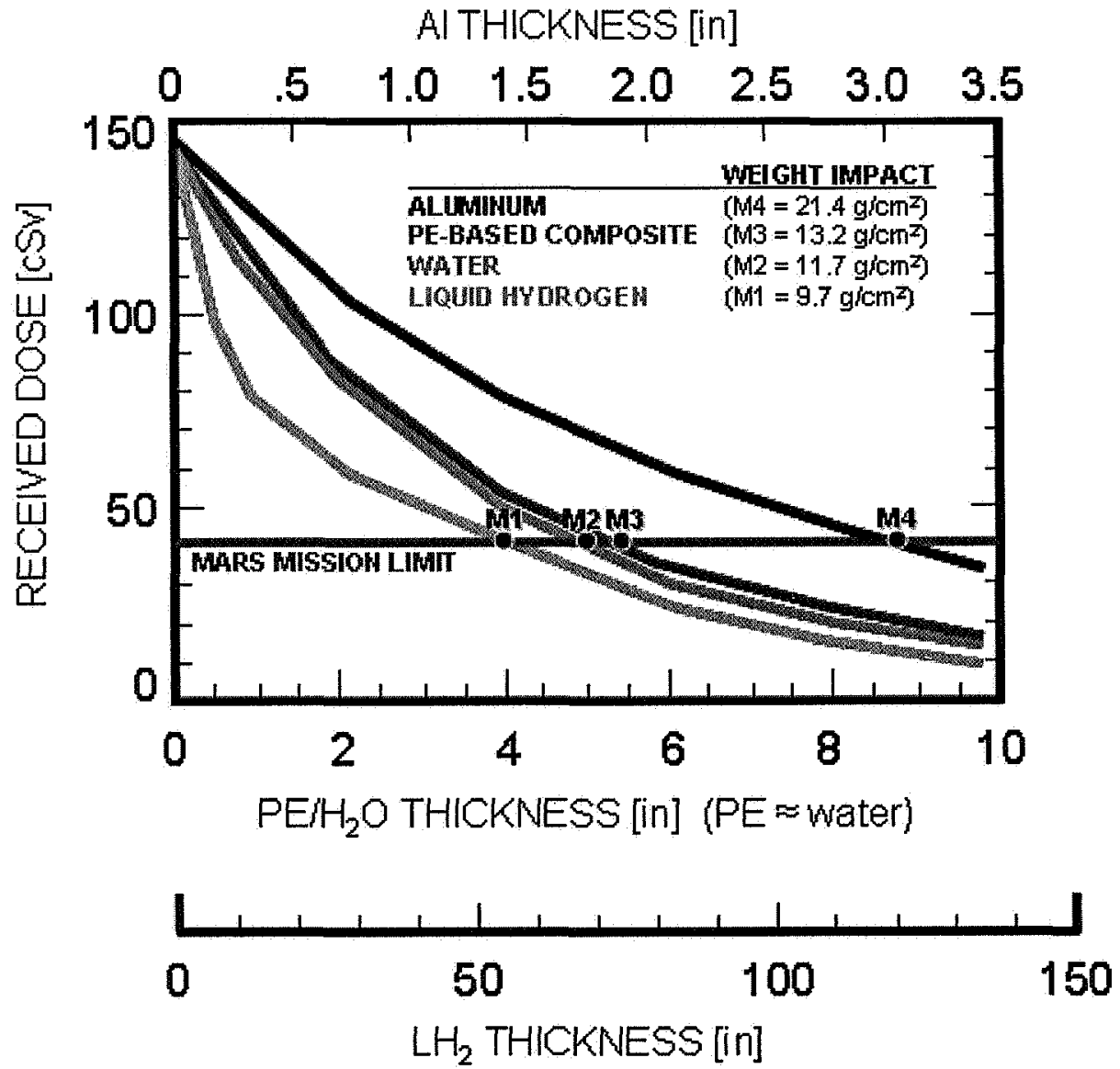

Figure 4 - Dose-equivalent as a function of depth in various materials. The assumed radiation environment is a hypothetical worst case scenario that superimposes solarminimum and a maximum solar particle event. This superposition is an overestimation of the radiation environment level for the sake of comparison. 
The scientific debate over uncertainty in biological data and risk methodologies has existed for decades ${ }^{6}$. These uncertainties cannot delay the functional optimization of shielding to degrade and/or deflect radiation as determined by experimental tests and the current empirical National Council on Radiation Protection \& Measurements (NCRP) established dose limitations for astronauts. Absent data from multiple, long duration manned missions and reliable predictions of future environments/events the uncertainties will remain.

As can be seen from top abscissa of the chart expressing thickness of conventional aluminum structural wall material, mass will become a very big factor during the development of these missions. It should be noted that proposals for new materials such as reinforced polyethylene composites can save as much as $40 \%$ over aluminum in aerial density of the structural wall based on dose, but even these new materials result in significant increases in mass of the structural wall.

As such, the NASA will have to learn how to utilize mass needed in the mission for other functions to help protect the crew from the harmful effects of the space radiation environment (e.g., vehicle cladding, propulsion expendables, environmental control and life support systems, thermal control systems, electronics, food, water, crew waste). Also, crew operations management will be a key to minimizing exposures requiring a new set of analytical skills and tools.

Based on today's knowledge, it is indicated that NASA must pursue multifunctional composites for the habitable volume of exploration vehicles not just for crew health, but for critical mass savings and protection of electrical circuitry.

\section{Fluids Distribution}

Thermal Control Systems (TCS), Environmental Control and Life Support Systems (ECLSS) including potable water, cooling water/fluids and waste products are required to be distributed throughout any spacecraft that is developed for exploration. Similar to radiation shielding, a FAST diagram can be constructed to facilitate multifunctional analysis. The current preferred method of fluids distribution is through conventional piping. However, a multifunctional approach suggests a cavity wall design where fluids are stored and transported within the space provided between structural wall materials or within "pipes" shaped from the core structural wall material. This blended architecture is driven in part by the necessity to locate fluids on the perimeter of the habitable volume to reduce radiation exposure and to eliminate the mass of a separate piping subsystem for each fluid.

Even for a theoretical closed loop ECLSS, waste will be generated and storage/disposal required. Due to re-supply limitations and contingencies, the reservoir of potable water will be much higher per astronaut than currently experienced on Space Station. Although certain gases may be expelled overboard, more research should be conducted in utilization of all wastes for densification of total perimeter acreage mass and other functional uses (e.g., methane). Also, ECLSS hardware must be developed to survive 
within the confines of the vacuum of a cavity wall design. There is little logic in necessitating a "shirt sleeve" environment for these components even if new thermal control methods must be developed.

New methods of circulating fluids and unique cold plate design embedded within the cavity wall will ensure thermal stability, eliminate stand-alone equipment and possibly eliminate valves. The development of radiators as part of the external cladding of the spacecraft can reduce the amount of support booms, additional piping and other hardware. Programmable sections of the internal cladding can be designed to coincidently remove heat as mounted equipment is utilized.

Fluids distribution incorporated into the design and development of a multi-functional structural wall system requires a new paradigm to look not just at the conventional requirements for these fluids around the spacecraft, but location consolidation, novel transport and substitute technologies. Physical architecture will play heavily on the hydraulics of fluid flow in microgravity, the multi-functional attributes of the fluid, volume considerations and the total mass impact of the design. Figure 5 illustrates schematically how water/wastewater could be integrated into a cross cutting cavity wall design.

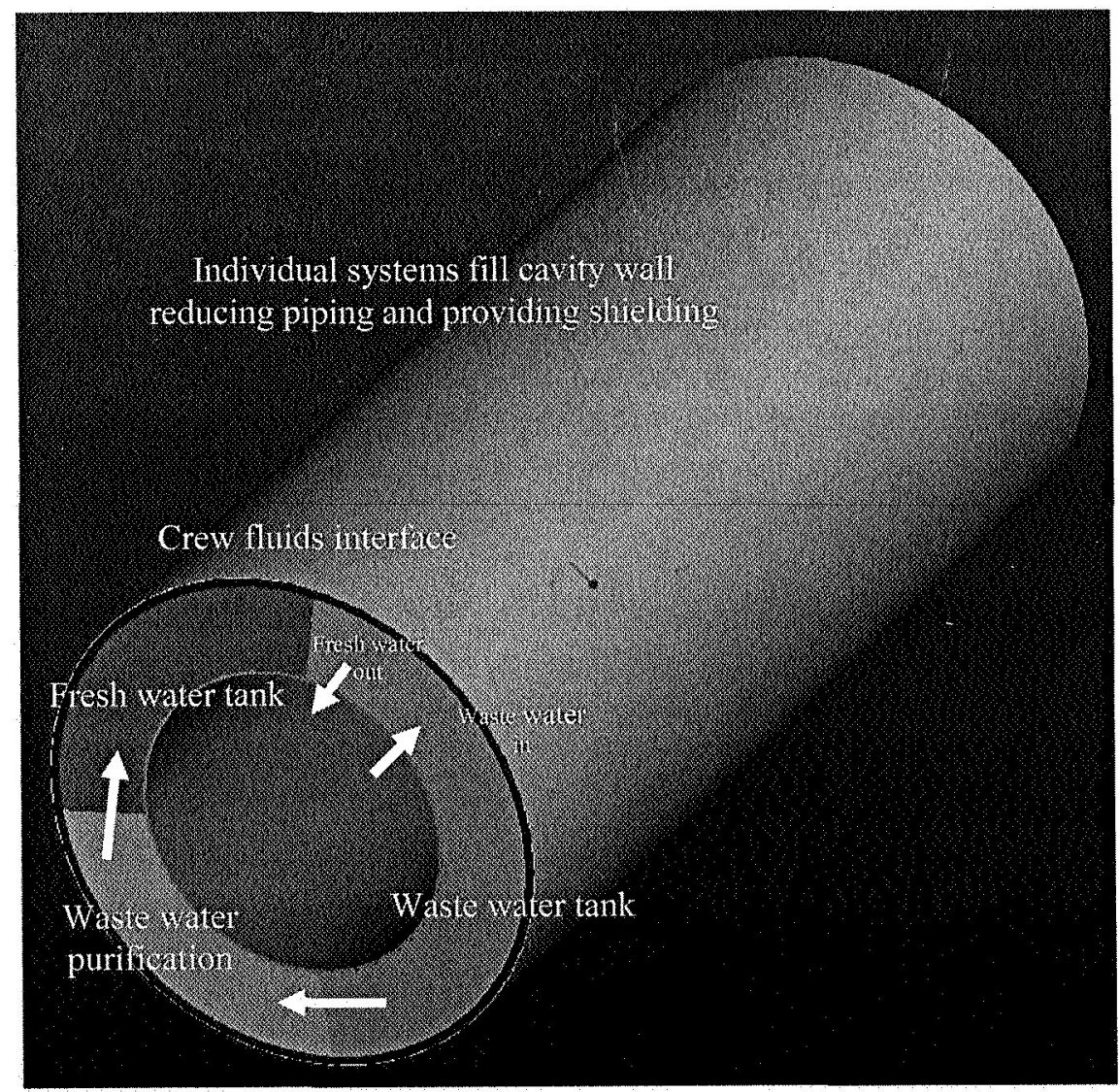

Figure 5 - Fluids Distribution Schematic 


\section{Electrical Distribution}

A Structurally Embedded Electrical Distribution (SEED) system is another example of FAST at work. The SEED is envisioned to optimize space requirements, provide robust spacecraft circuitry and reduce maintenance and repair costs. This innovation is a redundant secondary power distribution unit and structurally embedded electrical distribution system capable of automatically re-energizing a circuit that has been shorted from multiple network centric pathways. Aircraft and spacecraft power distribution systems typically consist of bundled insulated conductors susceptible to fraying, sharp corners, in-service damage and unforeseen impacts. Although redundant circuits can be created with such conventional distribution systems, they lack a multiplicity of redundancy, require "smart" switching devices, can be costly and remain susceptible to in-service damage, sharp corners and fraying.

Spacecraft composite structures such as fuselages, monocoque shells, multi-layered and stiffened panel designs introduce new architectural options that can produce robust vehicle systems. The nature of Polymeric Matrix Composites (PMC) manufacturing and especially the lay-up process lend itself to the development of multifunctional structures. The SEED introduces power distribution functions into the basic vehicle structure. Conductors such as copper, aluminum, carbon fiber and other suitable materials are introduced in between layers of "wet" pre-pregs (matrix pre-impregnated fiber layers) in specialized patterns that disperse redundant circuits geographically and in layers.

These circuits are then energized by conventional means either through grounded direct current or alternating current methods resulting in multiple access points (MAPs) for powered system subcomponents to be energized. The MAPs are installed in various locations around the vehicle or product depending on design. The MAP can be installed prior to or after autoclave curing dependent upon the matrix materials selected. The key characteristic of the MAP will be the functionality of the "pin-head contact" contained within the terminal secondary power distribution unit (TSPDU). The TSPDU is mounted onto the MAP after curing.

The automated, multiple redundancy of this network based power distribution system, its MAP and TSPDU are not known to exist. Although very conceptual in nature, the functional analysis associated with these type developments can drive many innovations. Such innovative processes as these may enable aerospace vehicles to sustain damage without losing power. The network distribution varies greatly with conventional bundled wire systems. This means of incorporating the wiring assembly into structures manufacturing eliminates individual conductor insulation, optimizes space requirements, decreases maintenance requirements and reduces risk consequences associated with short circuits or damage.

\section{Concept \& Iterative Design}

The combined attributes of mass, volume, power and radiation shielding for long duration missions when applied to a functional analysis of the single most fundamental system for manned spaceflight, the pressure wall, may drive the need for cavity wall architecture. Technologies and algorithms must be developed that will tailor the detailed disciplined 
design to a solution that meets each spacecraft's mission unique characteristics. Technology needs can be generated during FAST implementation (See Example in Appendix). Inherent in this cavity wall design will be:

- Advanced multi-functional materials

- Embedded subsystems

- Innovative low profile subsystems hardware

- Unique applications of vacuum based thermal protection

- Reduction of ECLSS, TCS \& power loads

- Elimination of secondary structure mass

- Innovative food storage techniques

- Simplification of heat removal systems

- Revolutionary depressurization/repressurization systems

- New solid human waste storage developments

- Flexible and modular architectural features for tailoring

- Scalability for earlier insertion in prototype vehicles

- Maximum system redundancy characteristics

The end result will realize a multi-functional cavity wall design with virtually every other spacecraft functional system incorporated to save mass, volume, power and maximize radiation shielding enabling long duration manned spaceflight anywhere and anytime. Figure 6 is an artistic rendering of the form of the cavity wall design.

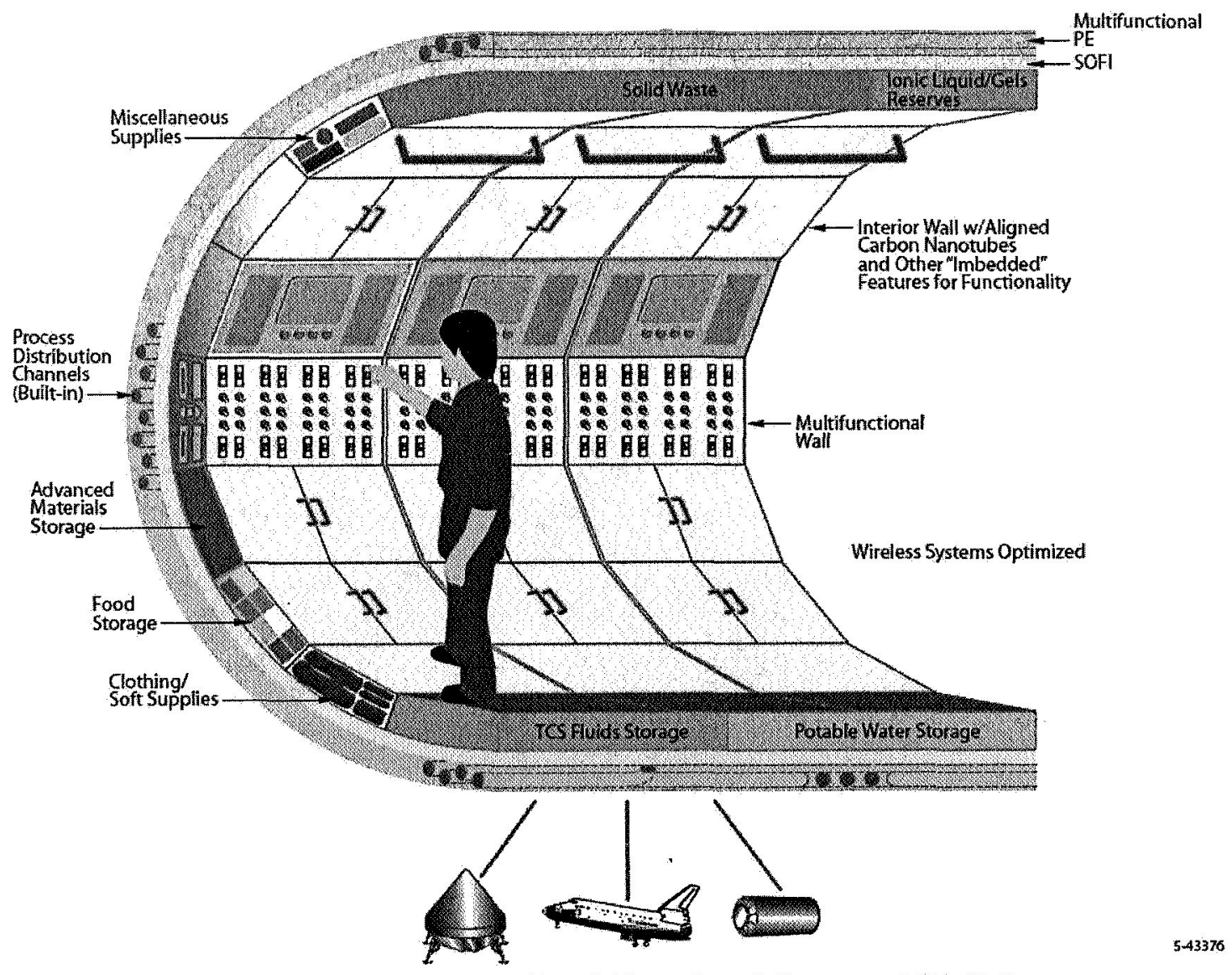

Figure 6 - Artist's Concept of Multifunctional Structural Wall System 
These structurally embedded systems can bring redundancy to some systems such as electrical, data and communications. Network oriented wiring, optical fibers and materially embedded communications systems will be developed in such a way that terminal application points can automatically reconfigure to ensure multiple pathways of functionality. Damage to portions of the distribution of these systems can easily be overcome due to the network deployment or reconfigured to allow architectural changes facilitating new functionality. Commonality and modularity of terminal point connections will not confine architectural changes to only the distribution avenues of the networked systems, but wholesale changes to accommodate mission altering conditions. This will be especially useful on long duration missions where all operational, environmental and contingency requirements cannot be fully incorporated in the final vehicle hardware design. The human space flight missions of the future will require maximum potential capabilities and flexibility to adapt. These crews will be very much capable of reconfiguring their spacecraft for optimal performance to meet the challenges in deep space far from any maintenance depot.

\section{Summary/Conclusion}

Cross cutting techniques in structural design is paramount in optimizing mass, volume, power and other key constraints in space exploration related product development. The foundational steps of structural design early in the conceptual phase must include these vigorous cross cutting techniques to achieve a robust system. The schedule pressures associated with the development phase do not present an environment wherein novel ideas, technology insertion, functional efficiencies or architectural changes are readily considered. The structural designer, as the first purveyor of physical definition, must be challenged constantly with more functional requirements by systems engineers. These challenges compel an environment of necessity that in turn drives the product and technology development processes.

Our eagerness to proceed with new developments must be tempered with basic fundamental functional analyses, tradeoffs, experience and heavy interdisciplinary dialogue to outline a product strategy inclusive of high priority technologies and evolution. This product strategy will have great influence on top level requirements, cost, schedule and the political realities of government programs. If done properly, the logical clarity of engineering analysis will contribute a more substantial role in decision making with regards to national space policy. Current trends do not bode well for the development of an environment characterized by strict engineering discipline, forward thinking, critical analyses and straightforward requirements driven development. The large dollar contracts, enormous workforce to transform from Shuttle and parochial politics will continue to press for point designs requiring future major modifications; compromises of function, modularity, efficiency and evolvability; and unfortunately bear actual costs way beyond any reasonable estimates. We must proactively show how iterative functional analyses can result in design synthesis capturing all requirements from policy objectives. 
In this paper we have emphasized the use of existing systems engineering and analytical techniques long espoused by the NASA community for the development of advanced exploration infrastructure. The FAST techniques illustrate how robust design solutions can be generated. The FAST techniques provide a foundational method for implementing and influencing national space exploration strategies including establishing technology priorities. While these techniques do not always result in validation of the various individual opinions of space infrastructure architecture, they fully encompass the tough environmental, operational and mission specific requirements of space exploration. Yes, there is a pathway to exploration and it is defined through a logical process of systems of systems engineering.

Absent a general concept for future spacecraft, some of the necessary technologies cannot be initiated and the stepwise path for long duration spaceflight may be delayed while these technologies are developed. Sharing the physical construct of many subsystems in a robust, multifunctional and embedded fashion within the confines of a pressure wall has not been accomplished previously for manned spacecraft. We showed that an architecture based on just that equipment directly interfacing with the astronaut within the "shirt sleeve" environment, results in many tactical advantages from a propulsion, power, ECLSS/TCS loading, shielding, volume and safety perspective. Developing these concepts from a cross cutting functional foundation is the key to a sustained path for the manned exploration of space. Simply speaking we must "begin with the end in mind" embracing the principles which have served our nation well in the past. ${ }^{8}$ 


\section{Appendix - An Example of Technology Requirements of a Multifunctional Structural Wall System}

Mechanical

- Valves

- Quick Disconnects

- Low Profile Enclosures

- ECLSS Redesign

- TCS Redesign

Sensor Development

- Embedded Designs

- Pressure/Temperature/Continuity

- Leak Detection/Isolation

- MCA/Particulate Contamination/Other

- Vehicle Health Monitoring

Multi-functional Materials

- System Analysis

- Composite Fabrication \& Testing

- Grounding \& Bonding

- Polymer Layering \& Integral LCD

Structural Modeling

- MMOD

- Ascent/Descent

- Aerocapture/Aeroassist

Fluids Flow Characterization

- Prototype Developments

- Ionic Liquids Research

Thermal Modeling

- Avionics

- ECLSS

- Greenhouse

- Food

- TCS

Compartment Access Design

- Vacuum Depress/Repress Mechanisms 


\section{Acknowledgements}

The author would like to acknowledge the assistance of Jeffrey L. Finckenor, Ted Allbritton, Chris Coppens, Paul Tatum, Nasser Barghouty for simulations of dose equivalents, peer reviewers and the support of the entire MSFC team.

\section{References}

1) Blair, J.C., Ryan, R.S., Schutzenhofer, L.A., Humphries, W.R., "Launch Vehicle Design Process: Characterization, Technical Integration, and Lessons Learned," NASA TP-2001-210992, May 2001.

2) Ryan, R.S., "The Role of Failure/Problems in Engineering: A Commentary on Failures Experienced - Lessons Learned," NASA TP-3213, March 1992.

3) George, T., Powers, R., "Closing the TRL Gap," Aerospace America, pgs. 24-26, August 2003.

4) Cook, S., Tyson R., "Next Generation Launch Technology Program Lessons Learned," AIAA Paper 2005-2562, $1^{\text {st }}$ Space Exploration Conference: Continuing the Voyage of Discovery, 30 January - 1 February 2005.

5) Stern, A., "Lunar and Mars Science and Exploration," MSFC Training Course, Slides 15 \& 16, Powerpoint file MSFC_Mars_Humans_to_Mars.ppt, July 2005.

6) Cucinotta, F.A., Kim, M.Y., Ren, L., "Managing Lunar and Mars Mission Radiation Risks Part I: Cancer Risks, Uncertainties, and Shielding Effectiveness," NASA TP-2005-213164, July 2005.

7) Garriott, O.K., Griffin, M., Claybaugh, W., Garvey, J., Jones, T.D., Kohlhase, C., McCandless, B., O'Neil, W., Penzo, P.A., "Extending the Human Presence into the Solar System," An Independent Study for the Planetary Society on Strategy for the Proposed U.S. Space Exploration Policy, July 2004.

8) Griffin, M.D., "Managing the Exploration of the Moon and Mars," Strategies for Mars: A Guide to Human Exploration, Stoker, C., Emmart, C., Eds., AAS Science and Technology Series Vol. 86, 1996.

9) Griffin, M.D., French, J.R., Space Vehicle Design, $2^{\text {nd }}$ Edition, AIAA, 2004.

10) Ball, J.R., Evans, C.H., Jr., "Safe Passage: Astronaut Care for Exploration Missions," Committee on Creating a Vision for Space Medicine During Travel Beyond Earth Orbit, Board on Health Sciences Policy, Institute of Medicine, National Academy Press, Washington, D.C., 2001.

11) Sarafin, T.P., Larson, W.J., Eds., Spacecraft Structures and Mechanisms - From Concept to Launch, Joint DoD/NASA Space Technology Series, 1995. 